\title{
Kinematics and helicity evolution of a loop-like eruptive prominence ${ }^{\star}$
}

\author{
K. Koleva ${ }^{1}$, M. S. Madjarska ${ }^{2}$, P. Duchlev ${ }^{1}$, C. J. Schrijver ${ }^{5}$, J.-C. Vial ${ }^{3,4}$, E. Buchlin ${ }^{3,4}$, and M. Dechev ${ }^{1}$ \\ 1 Institute of Astronomy and National Astronomical Observatory, Bulgarian Academy of Sciences, 72 Tsarigradsko Chaussee Blvd., \\ 1784 Sofia, Bulgaria \\ e-mail: koleva@astro.bas.bg \\ 2 Armagh Observatory, College Hill, Armagh BT61 9DG, N. Ireland \\ 3 CNRS, Institut d'Astrophysique Spatiale, UMR 8617, 91405 Orsay, France \\ 4 Univ Paris-Sud, Institut d'Astrophysique Spatiale, UMR 8617, 91405 Orsay, France \\ 5 Solar and Astrophysics Lab., Lockheed Martin Advanced Techn. Ctr., 3251 Hanover St., Bldg. 252, Palo Alto, \\ CA 94304-1191, USA
}

Received 5 December 2011 / Accepted 12 February 2012

\begin{abstract}
Aims. We aim at investigating the morphology as well as kinematic and helicity evolution of a loop-like prominence during its eruption.

Methods. We used multi-instrument observations from AIA/SDO, EUVI/STEREO and LASCO/SoHO. The kinematic, morphological, geometrical, and helicity evolution of a loop-like eruptive prominence were studied in the context of the magnetic flux rope model of solar prominences.

Results. The prominence eruption evolved as a height-expanding twisted loop with both legs anchored in the chromosphere of a plage area. The eruption process consisted of a prominence activation, acceleration, and a phase of constant velocity. The prominence body was composed of counter-clockwise twisted threads around the main prominence axis. The twist during the eruption was estimated at $6 \pi$ (3 turns). The prominence reached a maximum height of $526 \mathrm{Mm}$ before contracting to its primary location and was partially reformed in the same place two days after the eruption. This ejection, however, triggered a coronal mass ejection (CME) observed in LASCO C2. The prominence was located in the northern periphery of the CME magnetic field configuration and, therefore, the background magnetic field was asymmetric with respect to the filament position. The physical conditions of the falling plasma blobs were analysed with respect to the prominence kinematics.

Conclusions. The same sign of the prominence body twist and writhe, as well as the amount of twisting above the critical value of $2 \pi$ after the activation phase indicate that possibly conditions for kink instability were present. No signature of magnetic reconnection was observed anywhere in the prominence body and its surroundings. The filament/prominence descent following the eruption and its partial reformation at the same place two days later suggest a confined type of eruption. The asymmetric background magnetic field possibly played an important role in the failed eruption.
\end{abstract}

Key words. Sun: activity - Sun: filaments, prominences - magnetic fields

\section{Introduction}

Prominence eruptions are large-scale eruptive phenomena that occur in the low solar atmosphere. Observations show that prominences display a wide range of eruptive activity. There are three types of prominence (filament) eruptions according to the observational definitions of Gilbert et al. (2007) based on the relation between the filament mass and corresponding supporting magnetic structure: full, partial, and failed (confined), of which the partial ones are the most complex. A full eruption occurs when the entire magnetic structure and the pre-eruptive prominence material are expelled into the heliosphere. If neither the filament mass, nor the supporting magnetic structure escape the solar gravitational field, it is a failed eruption. Partial eruptions can be divided into two subcategories: i) when the entire magnetic structure erupts, with the eruption containing either part or none of its supported pre-eruptive prominence material; and ii) when the magnetic structure itself partially escapes with either

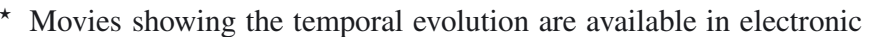
form at http: //www . aanda. org
}

some or none of the filament mass (Gilbert et al. 2007). One important observational consequence concerning partial and failed eruptions is the re-formation of the filament at the pre-eruptive location.

Sterling \& Moore (2004a,b) unveiled a common pattern of prominence eruptions: an initial slow-rise phase (with very low acceleration), during which the filament gradually ascends, followed by a sharp change to a phase of fast acceleration. There are three types of prominence eruption after the fast-rise phase: i) an eruptive prominence can continue to rise with acceleration; ii) the fast rise can be followed by a constant velocity phase; or iii) the constant velocity phase of an eruptive prominence can be followed by a deceleration phase (Vršnak 1998).

Eruptive prominences (EPs) (or filaments, if observed on the solar disk) are frequently associated with and physically related to coronal mass ejections (CMEs) and flares (TandbergHanssen 1995; Webb et al. 1976; Munro et al. 1979; Webb \& Hundhausen 1987; St. Cyr \& Webb 1991). Usually, all three eruptive events occur in the same large-scale coronal magnetic field, in which the EP only occupies a limited volume at its 
base. This association suggests that possibly the same physical process drives these eruptive phenomena. Filament-CME relationship studies often do not take into account the relation between filament-related magnetic field configurations, such as an empty filament channel, and a CME initiation (Alexander 2006). Therefore, the filament-CME relationship can be even stronger than is known so far. An EP and/or a CME are considered as the eruption of a preexisting magnetic flux rope (MFR) or an initial magnetic arcade that evolves into a magnetic flux rope during the eruption process via magnetic reconnection (Chen et al. 2006; Antiochos et al. 1999). However, the question whether an MFR exists prior to the eruption or is formed during the eruption remains controversial, with the MFR topology preceding the eruption being favoured in many studies during the last decade.

During activation, the slow rise motion of an EP is usually accompanied by a gradual morphological evolution from an initially intricate structure into an apparently toroidal shape, exposing sometimes a twisted pattern that is most often prominent in the legs of the prominence (Vršnak et al. 1991). Eruptive prominences very often develop a clearly helical shape in the course of the eruption, which is the characteristic signature of a magnetohydrodynamic (MHD) kink instability of a twisted MFR (e.g. Rust \& LaBonte 2005). An MFR becomes kink-unstable if the twist exceeds a critical value of $2 \pi$ (e.g. Hood \& Priest 1981 ; Fan 2005; Török \& Kliem 2005). The axis of an MFR then undergoes writhing (kinking) motions and part of the twist of the field is transformed into helical writhe of the axis, because the magnetic helicity is conserved (Rust \& LaBonte 2005). The conservation of helicity in ideal MHD (Berger 1984) requires the resulting writhe to be of the same sign as the transformed twist. The kink instability has long been investigated as a possible triggering mechanism for solar eruptive phenomena, especially in flux rope models (Liu \& Alexander 2009).

Kink and torus instabilities are suggested to be two mechanisms for triggering solar flares and coronal mass ejections (Sakurai 1976; Török \& Kliem 2005; Kliem \& Török 2006; Schrijver et al. 2008). Kink instability was first suggested as the trigger of prominence eruptions (confined and ejective) by Sakurai (1976) but has been generally regarded as a possible explanation only for confined events (e.g. Gerrard \& Hood 2003). Török \& Kliem (2005) and Fan (2005) succeeded in modelling the full ejection of a coronal flux rope from the Sun driven by kink instability. A kinking may be an important factor in the erupting process, but the type of eruption may strongly depend on the role played by magnetic reconnection and its location with regard to the prominence body (Antiochos et al. 1999). As discussed in Gilbert et al. (2007, and the references therein), the kink instability as a main trigger of prominence destabilisation and eruption is challenging to prove observationally because the helicity can force a flux rope to writhe without any instability occurring.

Kliem \& Török (2006) studied the expansion instability of a toroidal current ring embedded in a low-beta magnetised plasma. Schrijver et al. (2008) analysed two near-limb filament destabilisations involved in CMEs. Numerical simulation of a torus instability showed a relatively close quantitative match of the observations, implying that these two cases are likely to be torus instability eruptions.

Observations of filament eruptions that strongly suggest a helical kink occurring in flux rope topology were presented by Williams et al. (2005) for a full eruption, by Zhou et al. (2006) and by Liu et al. (2007) for partial eruptions, and Ji et al. (2003) and Alexander et al. (2006) for confined (failed) eruption. An observational definition of kinking related to the different types of filament eruptions is given by Gilbert et al. (2007) and Green et al. (2007).

In this paper, we investigate the phenomenology of an eruptive loop-shaped helically twisted prominence with fixed footpoints using state-of-the-art observations from the Atmospheric Imaging Assembly (AIA) onboard the Solar Dynamics Observatory (SDO) in the $304 \AA$ EUV passband. We have the unique opportunity to combine limb with on-disk observations of an eruptive prominence thanks to the EUVI/STEREO $\mathrm{B}$ observations, which at the time of the observations was at an angular distance of 71 degrees with the Earth. We aim at investigating the morphology as well as kinematic and helicity evolution of a loop-like prominence during its eruption. In Sect. 2 we describe the set of observations used in this study. In Sect. 3 we present the main results, which are discussed in Sect. 4. The conclusions are drawn in Sect. 5.

\section{Observations and data analysis}

The prominence eruption occurred at the north-east solar limb between 17:30 UT and 19:30 UT on 2010 March 30. The EP was centred at mean heliographic co-ordinates $\mathrm{N} 22.63^{\circ}$; E78.80 ${ }^{\circ}$ and mean position angle $66^{\circ}$.

For the present study we used images taken with $1 \mathrm{~min}$ cadence in the He II $304 \AA$ passband of AIA/SDO (AIA; Lemen et al. 2011). The AIA consists of seven Extreme Ultra-Violet (EUV) and three Ultra-Violet (UV) channels, which provide an unprecedented view of the solar corona with an average cadence of $\sim 12 \mathrm{~s}$. The AIA image field-of-view reaches 1.3 solar radii with a spatial resolution of $\sim 1.5^{\prime \prime}$. We used level 1 reduced data, i.e. with the dark current removed and the flat-field correction applied. The images were further stabilised for the satellite movements by applying intensity cross-correlation analysis using the SolarSoftware procedure get_correl_offsets.pro. For the purpose of our analysis we defined the visible limb from the AIA He II $304 \AA$ images. Note that the jitter correction was only needed this early in the mission because the image stabilisation was still subject to calibration in the commonissioning phase when these observations were taken.

We also analysed observations from the Extreme Ultraviolet Imager (EUVI) onboard STEREO Behind (B) spacecraft. EUVI has a field-of-view of $1.7 R_{\odot}$ and observes in four spectral channels (He II $304 \AA$ A, Fe IX/x $171 \AA$ A, Fe XII $195 \AA$ A and Fe XIV $284 \AA$ ) that cover the 0.1 to $20 \mathrm{MK}$ temperature range (Wuelser et al. 2004). The EUVI detector has $2048 \times 2048$ pixels $^{2}$ size and a pixel size of $1.6^{\prime \prime}$. We used images in the He II $304 \AA$ channel with an average cadence of 10 min. Images obtained by the Large Angle and Spectrometric Coronagraph (LASCO)/C2 onboard SOHO, whose field-of-view extends from 2 to 6 solar radii (Brueckner et al. 1995) were also analysed.

\section{Results}

\subsection{Morphology and helicity evolution}

The prominence eruption evolved as a height-expanding twisted loop with both legs anchored in the chromosphere of a plage area. The prominence before the activation can already be seen in $\mathrm{H} \alpha$ at 17:19 UT (Fig. 1). It shows as a few bright clouds above the limb with no distinguishable fine structure. In the AIA He II $304 \AA$ images at 17:32 UT and 17:40 UT in Fig. 2 (see also the online material), the fine structure of the prominence appears to be very dense, the prominence lies very close to the limb and, 
Table 1. The kinematics of the eruptive prominence derived from the AIA images.

\begin{tabular}{lcccc}
\hline \hline Phase & Time $(\mathrm{UT})$ & Height $(\mathrm{Mm})$ & Velocity $\left(\mathrm{km} \mathrm{s}^{-1}\right)$ & Acceleration $\left(\mathrm{m} \mathrm{s}^{-2}\right)$ \\
\hline Activation & $17: 33-18: 00$ & $18-34$ & 10 & 0 \\
Acceleration & $18: 00-18: 12$ & $34-121$ & $15-166$ & $46-430$ \\
Constant velocity & $18: 12-18: 44$ & $121-295$ & 91 & 0 \\
\hline
\end{tabular}

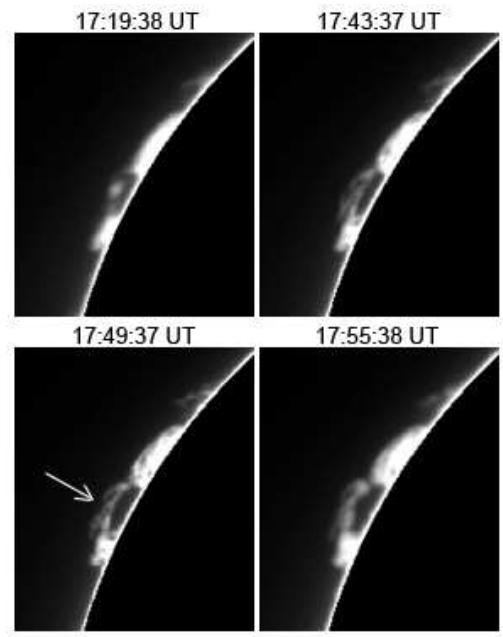

Fig. 1. Mauna Loa $\mathrm{H} \alpha$ images before and during the start of the prominence activation. The arrow points at the region revealing twisted structure.

therefore, it is impossible to distinguish its small-scale structure. Note that in $\mathrm{H} \alpha$ we see the cold core of the prominence, while in He II $304 \AA$ it is the envelope at higher temperatures (Labrosse et al. 2010). The $\mathrm{H} \alpha$ image at 17:49 UT shows the first signature of a twisted prominence fine structure. From part of the prominence where the fine structure is visible, we were able to estimate a $2 \pi$ twist, i.e. one turn of the prominence rope around its main axis. This time coincides with the beginning of the prominence's slow rise during the activation phase. The full amount of the prominence twist is first seen at around 18:20 UT (Fig. 2) in the AIA He II $304 \AA$ images. We estimated from the AIA $304 \AA$ images at 18:20 UT, 18:26 UT and 18:32 UT, a total twist of about $6 \pi$ ( 3 turns) of the eruptive prominence body.

We also searched for a signature of magnetic reconnection in all available data including AIA and EUVI He II $304 \AA$ and Fe XII $195 \AA$. Neither the off-limb AIA nor the on-disk EUVI data show any brightening in the footpoints of the prominence, their surroundings or along the prominence body. This suggests that magnetic reconnection may not have contributed to the prominence destabilisation and eruption.

After 18:25 UT the eruption can also be followed on the EUVI/STEREO image differences shown in Fig. 3 (see also the online material). Here, thanks to the different view point (see the AIA view translated on to the EUVI B image in Fig. 4) we were able to see that the filament upper body underwent a counter-clockwise writhing motion. The deformation of the prominence is still not visible in the AIA images at this time (Fig. 2) due to the line-of-sight effect. At 18:56 UT the crossing of the prominence body axis owing to the writhe as seen in projection on the disk in the STEREO difference images (Fig. 3) is visible at approximately half of the rising filament height. At 19:06 UT, this crossing is visible at approximately one third up from the feet of the prominence. The rotation angle increased with time, reaching $\pi / 2$ at 19:26 UT (Fig. 3).
The AIA He II $304 \AA$ images after 18:26 UT reveal best the helical twist of the prominence and its evolution in time (last row of Fig. 2). We clearly see that during the prominence uplift the twist transfers from the lower (legs) to the upper prominence body causing the prominence to evolve from a loop-shaped into a ribbon-like structure. With the help of the EUVI B difference images (Fig. 3), we established the footpoint location noted with black circles in Fig. 4 (top left). The so-defined foot positions are also shown in the EUVI image at 18:36 UT (Fig. 4, top right). A feature above the northern leg is also encircled and labelled with " $R$ ". This feature is used as a reference for the translation of the AIA images into the STEREO B view. In Fig. 4 (bottom right) we show the AIA $304 \AA$ image at 18:32 UT with the expanding loop-like prominence. This image was transformed into the STEREO B view and the legs of the prominence identified in the EUVI B images were transposed (shown with black circles), as was the reference feature. We paid special attention to the localisation of the feet of the EP to determine the type of twist and writhe, and their evolution in time. Based on the magnetic field polarity configuration obtained from the magnetograms, the $\mathrm{H} \alpha$ images and the prominence drawings (Fig. 5), we were able to determine in which magnetic polarity the southern and the northern legs of the prominence were located. From all the above information we established that the prominence underwent a writhe as a result of counter-clockwise rotation with respect to the two polarity fluxes.

\subsection{Kinematics}

The prominence height was determined as the height of the main axis of the prominence above the visible limb as observed in the He II $304 \AA$ channel of the AIA/SDO images (Fig. 6). The time evolution of the height reveals three distinctive phases of the prominence eruption: a prominence activation, an eruption with acceleration, and an eruption with a constant velocity (Fig. 7). From the first and second derivatives of the polynomial fit of the height-time curve, we defined the speed and the acceleration of the prominence eruption. The prominence activation is already in progress at the begining of the AIA observations around 17:33 UT. It is defined as the time period of a slow rise of the loop system with a velocity of $10 \mathrm{~km} \mathrm{~s}^{-1}$. Until 18:00 UT the height of the prominence changed from $18 \mathrm{Mm}$ to $34 \mathrm{Mm}$. The eruption onset was registered at 18:00 UT and it was determined from the AIA images as a sudden increase of the prominence height. It lasted until around 18:12 UT (Fig. 7). During this phase the prominence height changed from $34 \mathrm{Mm}$ to $121 \mathrm{Mm}$ and the speed of the prominence rise increased from $15 \mathrm{~km} \mathrm{~s}^{-1}$ to a maximum of $166 \mathrm{~km} \mathrm{~s}^{-1}$ with an acceleration from 46 to $430 \mathrm{~m} \mathrm{~s}^{-2}$. The constant velocity phase was measured until 18:44 UT. After this time the top of the looped prominence is outside the AIA field-of-view. The kinematics of the different phases of the eruptive process is summarised in Table 1.

It took the prominence $80 \mathrm{~min}$ to reach its maximum height (Fig. 3) at 19:16 UT as seen in the EUVI B images (note that the top of the prominence quits the AIA FOV after 18:44 UT). We were able to measure a descent of the prominence body with a 

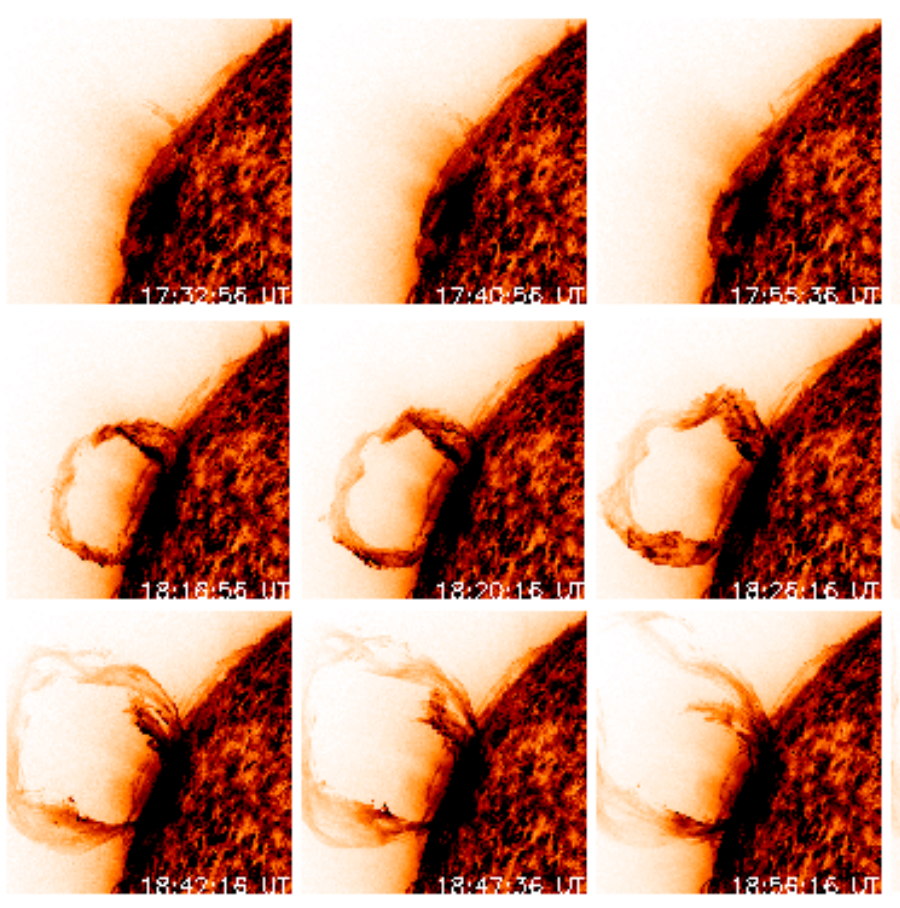

velocity of $\sim 50 \mathrm{~km} \mathrm{~s}^{-1}$ using three EUVI images, in which the prominence is clearly visible above the limb, i.e. at 19:16 UT, 19:26 UT and 19:36 UT (last row of Fig. 3).

After 18:47 UT (Fig. 2) the prominence legs are no longer twisted and we can clearly observe the downflow of individual plasma blobs thanks to the high AIA cadence of $1 \mathrm{~min}$ and its excellent spatial resolution. From the measurements of four blobs we found an average downflow speed of $31.8 \mathrm{~km} \mathrm{~s}^{-1}$ with a minimum of $13.7 \mathrm{~km} \mathrm{~s}^{-1}$ and maximum of $60 \mathrm{~km} \mathrm{~s}^{-1}$. Because of the continuous draining of the plasma towards the chromosphere, the prominence plasma density becomes lower and the prominence fainter in the AIA and EUVI B He II $304 \AA$ A images.

\subsection{A CME association}

At 18:30 UT C2/LASCO registered a CME at the eastern limb, which was associated with the eruptive prominence. The width of the CME was $64^{\circ}$ and the position angle was $74^{\circ}$. The prominence is located at the northern periphery of the CME magnetic system, at $19^{\circ}$ to the north of the position angle of the highest part of the CME. The CME propagated into the C2/LASCO field-of-view with a velocity decreasing from $853 \mathrm{~km} \mathrm{~s}^{-1}$ to $599 \mathrm{~km} \mathrm{~s}^{-1}$, implying a deceleration of $16.4 \mathrm{~m} \mathrm{~s}^{-2}$. The average linear velocity was $724 \mathrm{~km} \mathrm{~s}^{-1}$. At the location of the prominence eruption an expanding loop with an empty cavity underneath, which is part of the CME system, is clearly visible in the C2/LASCO images (indicated with an arrow in Fig. 8). Assuming a constant velocity of $91 \mathrm{~km} \mathrm{~s}^{-1}$ for the prominence eruption, at 18:54 UT, the prominence had reached a height of $390 \mathrm{Mm}$, while at 19:16 UT, when it reached its maximum height, it was at $526 \mathrm{Mm}$ (less than one solar radius). This strongly supports the observation that no prominence material is seen in the C2/LASCO FOV.

\subsection{Prominence reformation}

The prominence partially reformed in the same place two days after its eruption. In Fig. 5 the re-formation of the filament is

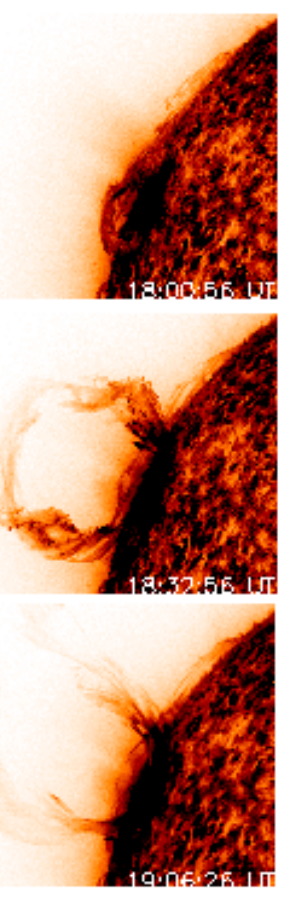

Fig. 2. He II $304 \AA$ AIA images (in reversed colour table) showing the morphology and, in particular, the helicity evolution of the erupting prominence. AIA He II $304 \AA$ image animation of the prominence eruption can be seen online.
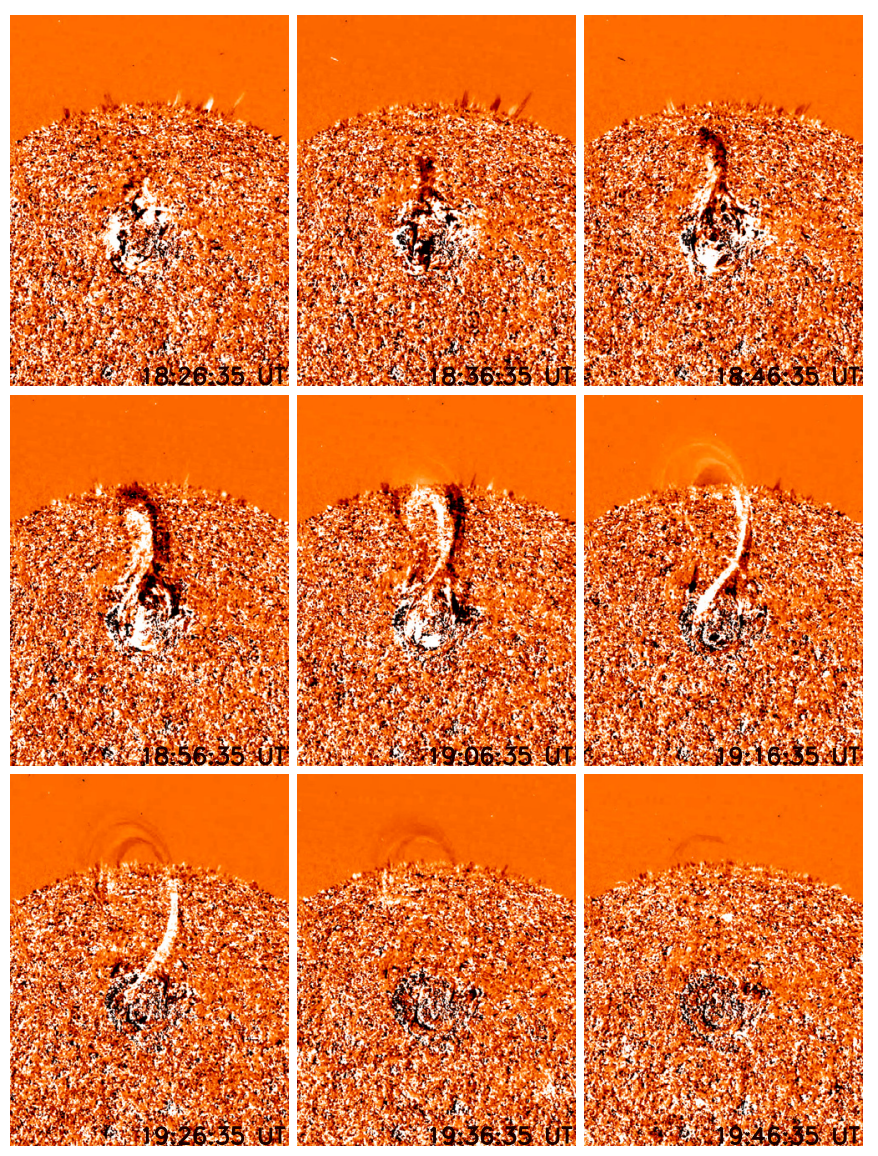

Fig. 3. Running difference images from EUVI B in the He II $304 \AA$ channel. EUVI/STEREO B He II $304 \AA$ A image difference animation of the prominence eruption on 2010 March 30 can be seen online.

presented from March 31 to April 2. On March 31, one day after its eruption, the filament can be traced only above and below the plage area on the Coimbra Solar Observatory $\mathrm{H}_{\alpha}$ (Fig. 5a). The 

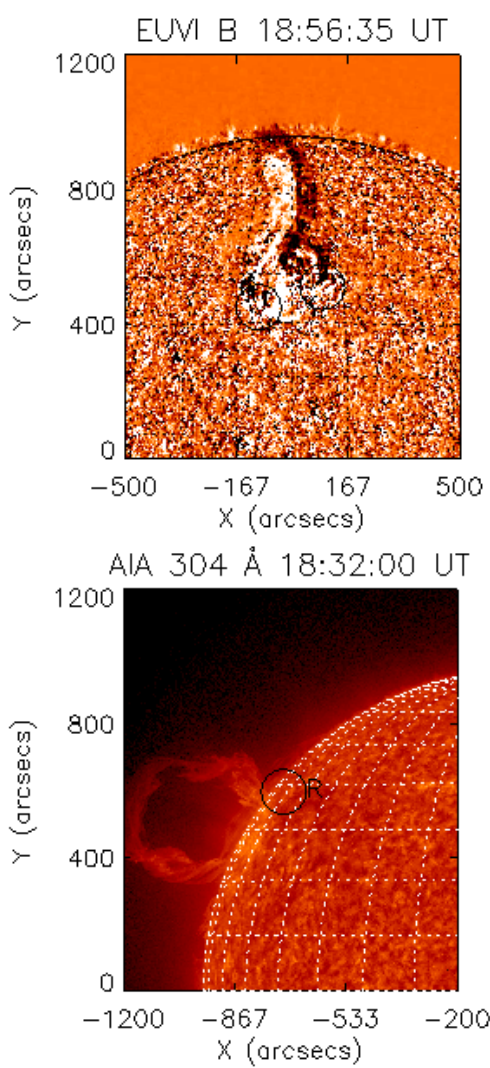
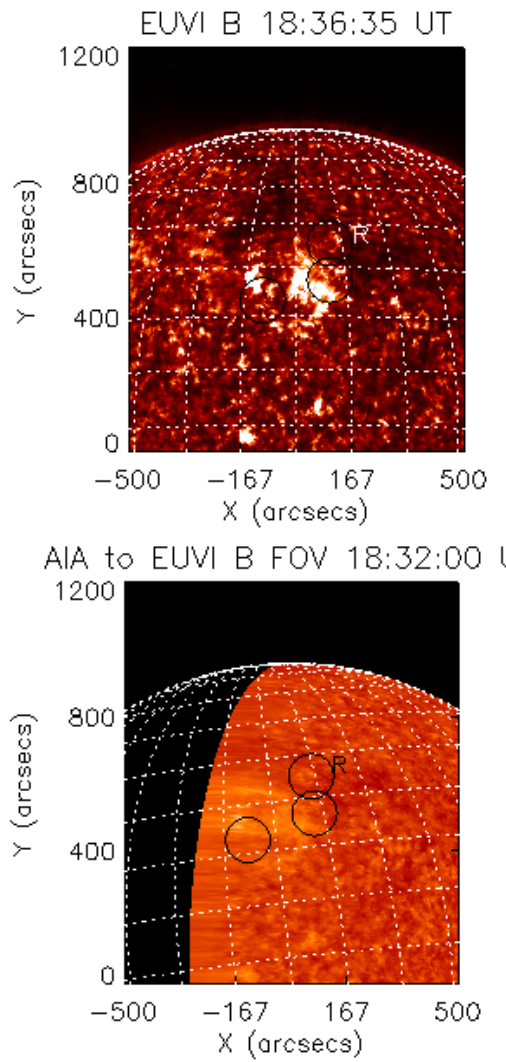

Fig. 4. EUVI difference image (top left) and EUVI image (top right). AIA image (bottom left) and the same image transformed to the STEREO FOV (bottom right). The filament's footpoints and a reference feature " $R$ " above the northern leg are marked with circles.
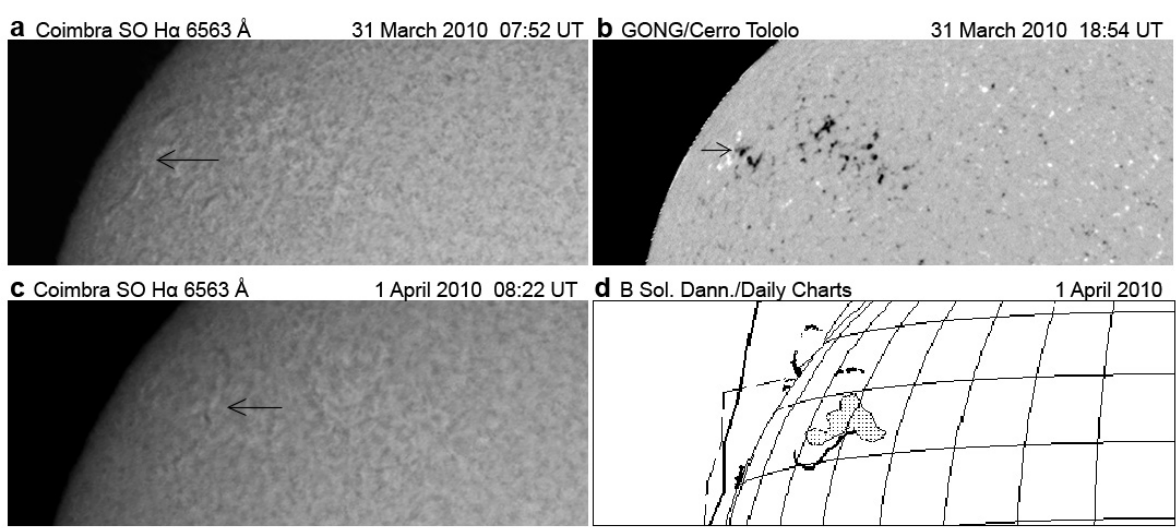

e Coimbra SO Ha $6563 \AA$ 2 April 2010 08:22 UT f B Sol. Dann.Daily Charts

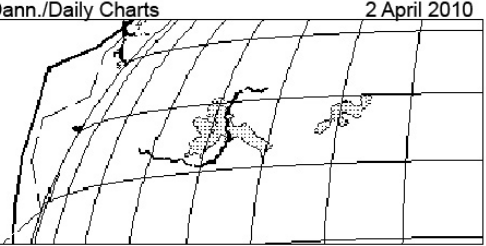

Fig. 5. Prominence reformation as seen on the disk as a filament traced in Coimbra Solar Observatory $\mathrm{H}_{\alpha}$ images (a), c), e)); a GONG magnetogram b); and daily drawings of the Sun from the electronic Bulletin "Solnechnye Dannye" of Pulkovo Observatory (d) and f)). magnetic field of the plage area associated with the reforming filament can be traced in Fig. 5b. Figures 5c-f show $\mathrm{H} \alpha$ images and drawings of the area from the Pulkovo Observatory, confirming the reformation of the filament. However, we can notice that the $\mathrm{H} \alpha$ filament is quite faint. We searched for its reformation in the EUV lines of EUVI B, but even in He II it was not possible to detect it. One explanation can be that some of the prominence material may have settled back to the photosphere because of the draining (Sect. 3.2), leaving the remaining material to settle in the magnetic skeleton of the prominence in the corona. Another possibility is that the material was not completely recovered at the low temperature typical of a filament.
As mentioned in Sect. 3.2, we analysed the kinematics of four blobs located at various altitudes in the EP. We were interested in connecting the prominence kinematics with the physical conditions in this falling material, which contributes to the reformation of the prominence. We computed the variation (with altitude or time) of the blob's He II $304 \AA$ emission with a careful subtraction of the (varying) background and a normalisation by the quiet-Sun He II intensity, known to be of the order of $8400 \mathrm{erg} \mathrm{s}^{-1} \mathrm{~cm}^{-2} \mathrm{sr}^{-1}$ (Vernazza \& Reeves 1978). The ratio $I$ (blob)/I(QS) was found to vary between 0.1 (mostly at high altitudes $100000 \mathrm{~km}$ ) to more than 1 (at low altitudes 

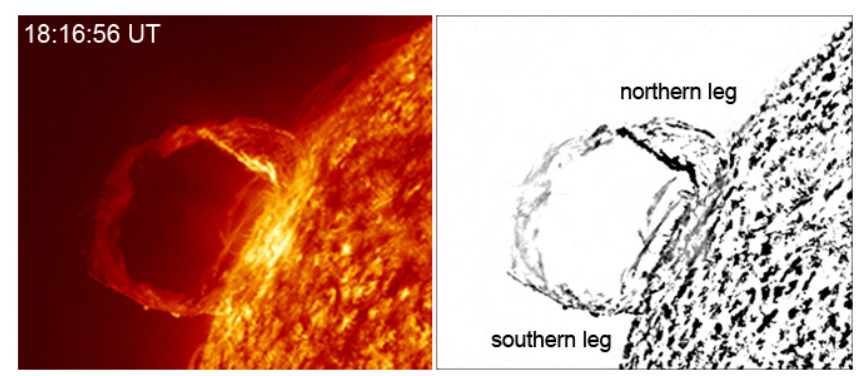

Fig. 6. Eruptive prominence on 2010 March 30 observed in the $304 \AA$ AIA/SDO channel at 18:17 UT (left) and its corresponding edgeenhanced image (right).

$20000 \mathrm{~km}$ ), without an evident trend with time. Although we do not have spectral information, one can derive some information from the non-LTE modelling, such as performed by Labrosse $\&$ Gouttebroze (2001) for prominences. Their range of temperatures is limited to $\sim 20000 \mathrm{~K}$ but their range of widths (up to $\sim 10000 \mathrm{~km}$ ) is well comparable to the size of our blobs (between 4000 and $8400 \mathrm{~km}$, assuming a spherical shape). From their Figs. 15 and 19, one can see that the He II $304 \AA$ intensity is of the order of $1000( \pm 200) \mathrm{erg} \mathrm{s}^{-1} \mathrm{~cm}^{-2} \mathrm{sr}^{-1}$, which implies a mean $I$ (prominence) $/ I(\mathrm{QS})$ ratio of 0.12 . From this result, one can derive that the $I(\mathrm{blob}) / I(\mathrm{QS})$ values found at high altitudes correspond to a low temperature, at least in the range of values (6000-20000 K) of Labrosse \& Gouttebroze (2001). The high values found at lower altitudes can be tentatively interpreted as the signature of much higher temperatures (corresponding to the usual $70000 \mathrm{~K}$ value).

\section{Discussion}

Although the velocity evolution profile of the prominence/filament analysed here is similar to torus-ring eruptions, a very clear twist of the prominence body above a critical value of $2 \pi$ that evolves into a writhe does favour the kink instability scenario. However, a kink instability may not have been the only trigger for this eruption as, for instance, the data do not reveal any indication of magnetic reconnection. A clear signature of the VERY triggering of destabilisation based on the present observations is, therefore, difficult to establish. Conclusive observations on the onset of prominence eruptions would require magnetic field measurements in the footpoints of eruptive prominences, combined with imaging and spectroscopic observations.

We examined the possible role of kink instability in the eruption of the prominence. A magnetic flux rope becomes kinkunstable if the twist of the magnetic field $T_{\mathrm{w}}$, a measure of the number of turns of the magnetic field lines about the prominence main axis, exceeds a critical value of $2 \pi$ (Hood \& Priest 1981). During the instability a flux tube would helically untwist, reducing the magnetic stress caused by this instability. The magnetic helicity, $H_{\mathrm{m}}$, can be expressed as the combination of twist and writhe, i.e. $H_{\mathrm{m}}=\Phi^{2}\left(T_{\mathrm{w}}+W_{\mathrm{r}}\right)$, where $\Phi$ is the axial magnetic flux in the flux rope and $W_{\mathrm{r}}$ is the measure of the twist of the rope around itself. Because of the conservation of magnetic helicity (Berger 1984), the twist will transfer into a writhe, $W_{\mathrm{r}}$, i.e. forming a ribbon-like structure. Vršnak et al. (1991) found that noneruptive prominences have a total twist less than $2 \pi$ (less than one complete turn) and, in contrast, all eruptive prominences that had a total twist $\geq 2.5 \pi$ (one and a quarter turns) erupted. On theoretical grounds Mikić et al. (1990) found that a purely

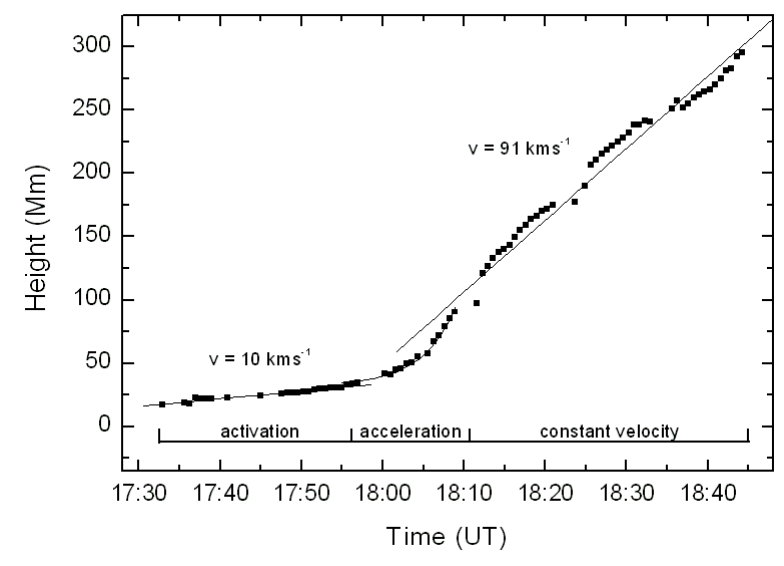

Fig. 7. Height-time profile of the prominence eruption for the three phases of the EP evolution: the activation, the acceleration, and the constant velocity.

axial loop that is twisted at its footpoints becomes kink-unstable when the twist exceeds $4.8 \pi$.

In the present case the prominence shows a counterclockwise twist of $6 \pi$, which transfers during the eruption from the prominence legs to its upper body, forming a counterclockwise writhe. This twist of the prominence body can be clearly seen on the AIA He II $304 \AA$ images, while the writhe of the eruptive filament is evident from the images of EUVI/STEREO B (Fig. 3). A flux rope helical twisting and writhing of the same sign is a basic condition for kink instability to work (Hood \& Priest 1979). That leads us to suggest that the eruptive prominence analysed here was possibly subjected to a kink instability, although other instabilities may also have played some role.

The eruptive prominence was located in the northern periphery of a large-scale CME magnetic configuration. Therefore, the background magnetic field is asymmetric with respect to the filament position. There are several observational (e.g. Ji et al. 2003; Alexander et al. 2006; Green et al. 2007; Liu et al. 2009; Shen et al. 2011) and theoretical (Török \& Kliem 2005; Kliem \& Török 2006; Fan \& Gibson 2007; Liu 2008) studies suggesting a confining role of the overlying magnetic field during failed filament eruptions owing to a slowly decreasing gradient of the magnetic field and a strong field intensity at low altitude. Recently, Liu et al. (2009) investigated a failed filament eruption, in which two filaments are asymmetrically located in coronal loops. The authors compared the confinement ability of symmetric and asymmetric fields and found that the magnetic confinement of an asymmetric field is stronger than that of a symmetric one. Shen et al. (2011) made these calculations for six EPs, five of which represented failed eruptions and one a successful eruption. The authors suggested that an asymmetric background field is an important factor that leads to failed filament eruptions.

\section{Conclusions}

We examined the kinematic and helicity pattern together with the morphological and geometrical evolution of an EP, based on He II $304 \AA$ AIA/SDO and EUVI/STEREO B observations. The unique combination of high-resolution limb observations of the EP in AIA and a central meridian position in EUVI B permitted a detailed analysis of the prominence eruption on 2010 March 30. The obtained results can be summarised as follows: 

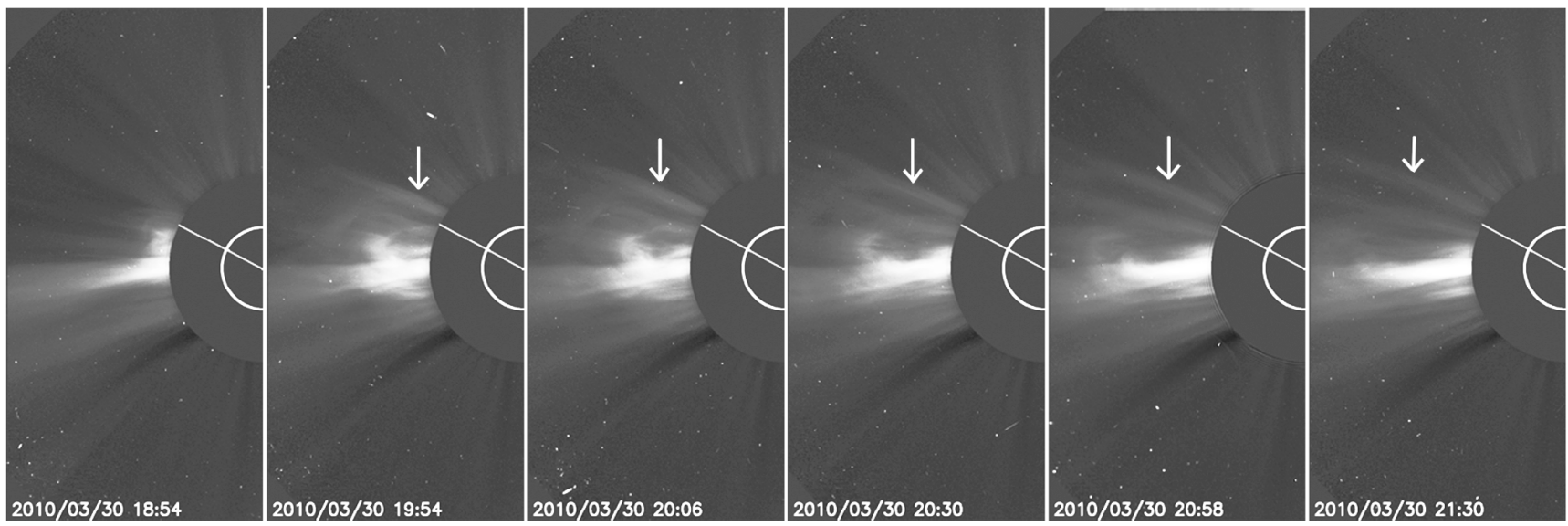

Fig. 8. SOHO/LASCO C2 registrations between 18:54 UT and 23:42 UT on 30 March 2010. The CME is first shown at 18:54 UT with a central position angle of $86^{\circ}$. The EP is located in the northern periphery of CME magnetic system. The white radial line over-plotted on the occulting disk indicates the central EP position angle $\left(55^{\circ}\right)$ at the eastern limb.

The EP appeared as a helically twisted MFR with fixed footpoints. The prominence's body was composed of counterclockwise twisted threads around the main prominence axis. The twist during the eruption was estimated to be $6 \pi$ (3 turns).

The EP twist progressively converted into a counterclockwise writhe that is clearly traced in the EUVI/STEREO $\mathrm{B}$ images. The position of the crossing-point of the writhed prominence body descended, while the eruption was progressing. Simultaneously the drain of prominence plasma towards the chromosphere can be followed in the AIA/SDO images.

The height-time profile of the EP revealed three distinctive phases of prominence eruption: a prominence activation phase, accelerating phase, and eruptive phase, the last one with constant velocity. Consequently, the prominence contracted to its primary location after reaching a maximum height of $526 \mathrm{Mm}$. The prominence/filament partially reformed, suggesting that either part of the material has drained into the photosphere or that not all of the prominence material returned to its primary temperature.

The draining plasm a blobs at low altitudes were found to have high temperatures, which could result from the encounter of the downflowing plasma with the static plasma in the legs and the resulting heating compression.

The EP was associated with a narrow expanding loop and an empty cavity underneath, located in the northern periphery of a CME, which appeared at the eastern limb and was triggered by the prominence eruption.

Acknowledgements. The authors thank the anonymous referee for the helpful suggestions and comments. The AIA data are courtesy of SDO (NASA) and the AIA consortium. Research at Armagh Observatory is grant-aided by the N. Ireland Department of Culture, Arts and Leisure. This work is grant-aided by the Bulgarian Academy of Sciences via the Institute of Astronomy. The authors thank the STEREO/SECCHI consortium for providing the data. The SECCHI data used here were produced by an international consortium of the Naval Research Laboratory (USA), Lockheed Martin Solar and Astrophysics Lab (USA), NASA Goddard Space Flight Center (USA), Rutherford Appleton Laboratory (UK), University of Birmingham (UK), Max-Planck-Institut for Solar System Research (Germany), Centre Spatiale de Liège (Belgium), Institut d'Optique Théorique et Appliquée (France), Institut d'Astrophysique Spatiale (France). J.C.V. and E.B. acknowledge the support of CNRS in the frame of the CNRS/BAS agreement (28 April 2010, \# 66459). They also acknowledge the support of CNES (Space French Agency) for easing the access to AIA/SDO data. They thank Claude Mercier for her continuous support.

\section{References}

Alexander, D. 2006, Space Sci. Rev., 123, 81

Alexander, D., Liu, R., \& Gilbert, H. R. 2006, ApJ, 653, 719

Antiochos, S. K., DeVore, C. R., \& Klimchuk, J. A. 1999, ApJ, 510, 485

Berger, M. A. 1984, Geophys. Astrophys. Fluid Dyn., 30, 79

Brueckner, G. E., Howard, R. A., Koomen, M. J., et al. 1995, Sol. Phys., 162, 357

Chen, J., Marqué, C., Vourlidas, A., Krall, J., \& Schuck, P. W. 2006, ApJ, 649, 452

Fan, Y. 2005, ApJ, 630, 543

Fan, Y., \& Gibson, S. E. 2007, ApJ, 668, 1232

Gerrard, C. L., \& Hood, A. W. 2003, Sol. Phys., 214, 151

Gilbert, H. R., Alexander, D., \& Liu, R. 2007, Sol. Phys., 245, 287

Green, L. M., Kliem, B., Török, T., van Driel-Gesztelyi, L., \& Attrill, G. D. R. 2007, Sol. Phys., 246, 365

Hood, A. W., \& Priest, E. R. 1981, Geophys. Astrophys. Fluid Dyn., 17, 297

Ji, H., Wang, H., Schmahl, E. J., Moon, Y.-J., \& Jiang, Y. 2003, ApJ, 595, L135

Kliem, B., \& Török, T. 2006, Phys. Rev. Lett., 96, 255002

Labrosse, N., \& Gouttebroze, P. 2001, A\&A, 380, 323

Labrosse, N., Heinzel, P., Vial, J.-C., et al. 2010, Space Sci. Rev., 151, 243

Lemen, J. R., Title, A. M., Akin, D. J., et al. 2011, Sol. Phys., 172

Liu, Y. 2008, ApJ, 679, L151

Liu, R., \& Alexander, D. 2009, ApJ, 697, 999

Liu, R., Alexander, D., \& Gilbert, H. R. 2007, ApJ, 661, 1260

Liu, Y., Su, J., Xu, Z., et al. 2009, ApJ, 696, L70

Mikić, Z., Schnack, D. D., \& van Hoven, G. 1990, ApJ, 361, 690

Munro, R. H., Gosling, J. T., Hildner, E., et al. 1979, Sol. Phys., 61, 201

Rust, D. M., \& LaBonte, B. J. 2005, ApJ, 622, L69

Sakurai, T. 1976, PASJ, 28, 177

Schrijver, C. J., Elmore, C., Kliem, B., Török, T., \& Title, A. M. 2008, ApJ, 674, 586

Shen, Y.-D., Liu, Y., \& Liu, R. 2011, Res. Astron. Astrophys., 11, 594

St. Cyr, O. C., \& Webb, D. F. 1991, Sol. Phys., 136, 379

Sterling, A. C., \& Moore, R. L. 2004a, ApJ, 602, 1024

Sterling, A. C., \& Moore, R. L. 2004b, ApJ, 613, 1221

Tandberg-Hanssen, E. 1995, The nature of solar prominences, Astrophys. Space Sci. Lib., 199

Török, T., \& Kliem, B. 2005, ApJ, 630, L97

Vernazza, J. E., \& Reeves, E. M. 1978, ApJS, 37, 485

Vršnak, B. 1998, in New Perspectives on Solar Prominences, ed. D. F. Webb,

B. Schmieder, \& D. M. Rust, IAU Colloq. 167, ASP Conf. Ser., 150, 302

Vršnak, B., Ruzdjak, V., \& Rompolt, B. 1991, Sol. Phys., 136, 151

Webb, D. F., \& Hundhausen, A. J. 1987, Sol. Phys., 108, 383

Webb, D. F., Krieger, A. S., \& Rust, D. M. 1976, Sol. Phys., 48, 159

Williams, D. R., Török, T., Démoulin, P., van Driel-Gesztelyi, L., \& Kliem, B. 2005, ApJ, 628, L163

Wuelser, J., Lemen, J. R., Tarbell, T. D., et al. 2004, in SPIE Conf. 5171, ed. S. Fineschi, \& M. A. Gummin, 111

Zhou, G. P., Wang, J. X., Zhang, J., et al. 2006, ApJ, 651, 1238 